\title{
Parathyroid lipoadenoma: a diagnostic pitfall during frozen section evaluation of parathyroid lesions
}

\begin{abstract}
Parathyroid lipoadenomas are a rare and benign variant of parathyroid adenomas that are defined morphologically by an abundance of adipocytes. The clinical manifestations and the laboratory findings are indistinguishable from those of the usual forms of parathyroid adenomas. Unlike parathyroid adenomas, parathyroid lipoadenomas can be functional or nonfunctional. We present a case of a functioning parathyroid lipoadenoma, which presented diagnostic difficulty on frozen section examination. The diagnosis was aided by intra-operative parathyroid hormone measurement.
\end{abstract}

Keywords: parathyroid; hyperparathyroidism, PTH, adenoma, parathyroid hormone, lipoadenoma, parathyroid lipoadenoma, PLA

\author{
Volume 3 Issue 2 - 2016
}

\author{
Kiran Krishne Gowda,' Prasanna Matippa ${ }^{2}$ \\ 'Department of Laboratory Medicine I, NU Hospitals, India \\ ${ }^{2}$ Department of Urology, NU Hospitals, India
}

Correspondence: Kiran Krishne Gowda, Consultant

Pathologist, NU Hospitals, Bangalore, India, Tel 9902313888, Email dr.k.kiran@nuhospitals.com

Received: October 12, 2016 | Published: November II, 2016

\section{Introduction}

Primary hyperparathyroidism is a disorder of inappropriate secretion of parathyroid hormone (PTH) from parathyroid tissue. Majority $(80 \%$ to $85 \%)$ of primary hyperparathyroidism can be attributed to single enlarged parathyroid gland or adenoma. ${ }^{1}$ Parathyroid lipoadenoma (PLA) is extremely rare cause of hyperparathyroidism and differs from typical adenoma with regards to 1 major histologic criterion: this histologic variant is defined by presence of abundant stromal fat. ${ }^{2}$ Approximately 60 cases of parathyroid lipoadenoma have been documented in English literature. ${ }^{3-7}$ We present a rare case of hyper-functioning lipoadenoma, diagnosis of which was aided by intra-operative PTH measurement.

\section{Case history}

A 36 year old man with history of recurrent right ureteric calculi since 3 years presented with left ureteric colic. Computed Tomography scan of abdomen revealed calculus in left upper ureter measuring $7 \times 4 \mathrm{~mm}$ and hydronephrosis of left kidney. His biochemical tests revealed serum calcium of $10.01 \mathrm{mg} / \mathrm{dL}$ (normal- 8.1 to $10.2 \mathrm{mg} / \mathrm{dL}$ ), and serum PTH of $257 \mathrm{pg} / \mathrm{mL}$ (normal- 14 to $72 \mathrm{pg} / \mathrm{mL}$ ). In view of hyperparathyroidism, Sestamibi scan was done, which revealed solitary hyper functioning right inferior parathyroid gland in right trachea-esophageal groove (Figure 1).

Suspecting an adenoma, right inferior parathyroid gland was excised along with left ureteroscopy and laser fragmentation of calculus. The parathyroid tissue sent to frozen section weighed $2000 \mathrm{mg}$ and measured $2.5 \times 1.5 \times 1 \mathrm{~cm}$ (Figure $2 \mathrm{~A}$ ). Frozen section examination confirmed parathyroid tissue showing glandular elements admixed with adipocytes. Parathyroid tissue was labelled abnormal (lipoadenoma/lipohyperplasia) in view of weight and measurement. Rapid intraoperative PTH levels guided surgical procedure. Preincision PTH was $350.4 \mathrm{pg} / \mathrm{mL}$, while post-excision PTH was $18 \mathrm{pg} /$ $\mathrm{mL}$ (20 minutes after excision). A significant decrease of intraoperative PTH level of $>50 \%$ from maximum baseline indicated successful excision of adenoma and negated need for further exploration.
A definitive diagnosis was established on histopathological examination of paraffin block of parathyroid tissue which revealed lipoadenoma with cords and nodular expansions of glandular elements (Figure 2) intimately admixed with adipocytes $(60 \%$ of tumor). Minimal nuclear atypia was noted. However, no necrosis, fibrous band, mitosis, capsular or vascular invasion was noted. A peripheral rim of normal parathyroid tissue was noted. Post-operatively patient had an uneventful recovery. At the time of last follow-up (6 months post surgery) his serum calcium was $8.9 \mathrm{mg} / \mathrm{dl}$ (normal: $8.5-10.1 \mathrm{mg} /$ dl) and PTH was $41.4 \mathrm{pg} / \mathrm{ml}$ (normal: $16-72 \mathrm{pg} / \mathrm{ml}$ ).

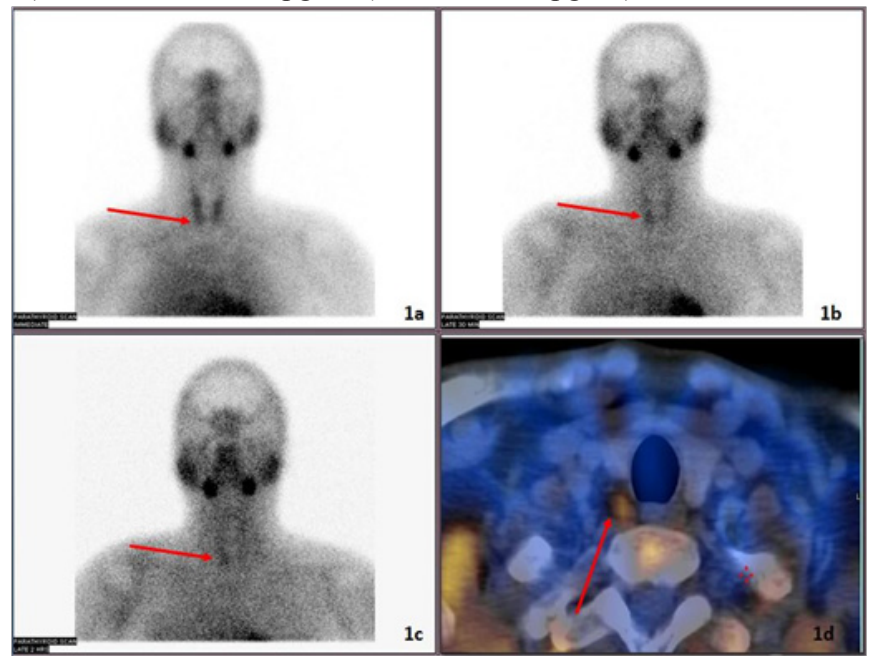

Figure I Planar sestamibi scan of the neck immediately (A), at 30 minutes (B) and at 2 hours (C) shows a persistent focal region of increased uptake separate from the thyroid gland at the level of the top of the sternum compatible with a parathyroid adenoma (arrows show the parathyroid adenoma; the thyroid gland fades on the 2 hour images). 3D-SPECT (single photon emission computed tomography) with sestamibi tracer axial image (D) depicts abnormal accumulation in the right trachea-esophageal groove behind the right lower pole region of thyroid gland (arrow). 


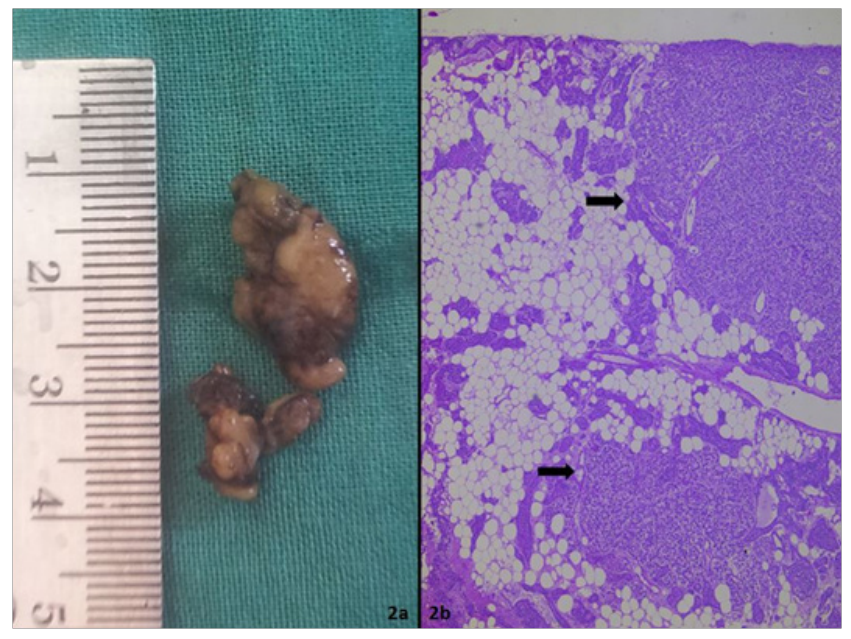

Figure 2 Panel of photograph shows gross (A) and microscopic images (B) of parathyroid lipoadenoma. Microscopy reveals approximately $50 \%$ adipose tissue separating cords and nodular expansions of oxyphil cells (arrows), characteristic of parathyroid lipoadenomas (Hematoxylin \& eosin stain; x40).

\section{Discussion}

PLA is extremely rare, constituting $<1 \%$ of all causes of hyperparathyroidism. ${ }^{3}$ It is a variant of adenoma characterized by abundance of adipocytes. Approximately 60cases of PLA have been documented in English literature. ${ }^{3-7}$ The current case would be $61^{\text {st }}$ case. Similar to tumor in the current study more than $50 \%$ of PLA documented are functional.

Fat normally occupies approximately $25 \%$ of normal parathyroid gland and it may be increased by advanced age and obesity. The fat content of PLA is highly variable. Chow et al documented $>50 \%$ fat in all 5 PLAs in their study. ${ }^{4}$ Seethala et al. ${ }^{3}$ reported a median fat content of $50 \%$ (range, $30 \%-70 \%$ ) in 8 lipoadenomas. It was $60 \%$ in the current case. The origin of fatty tissue component remains unknown, but it has been speculated that same factors that drive the enlargement of parathyroid chief cells are responsible for enlargement of fatty component. The high fat content of lipoadenomas renders them difficult to localize using imaging techniques. Ultrasound has been reported to identify the tumors in $50 \%$ of cases, whereas sestamibi was successful in $71 \%$ in a series of 11 cases. $^{3}$ In our patient, sestamibi single photon emission computed tomography images showed increased activity in the right inferior parathyroid gland.

Lipoadenomas resemble normal parathyroid tissue because of their abundant stromal fat, which may cause diagnostic difficulty at the time of frozen section. Seethala et al evaluated the performance of frozen section in this setting and found that in all cases where frozen sections were performed, they were able to successfully identify that the parathyroid tissue removed was abnormal. ${ }^{3}$ They attributed their success largely to accurate measurement, weight, and good communication between the surgeon and pathologist as to how much of the gland was resected. This was the case in our patient. The parathyroid gland was labelled abnormal on frozen section, based on measurement, weight and complete excision.

Intraoperative PTH measurement is extremely useful in assessing the adequacy of excision of parathyroid adenoma. A significant decrease of the intraoperative PTH confirms that further exploration is not indicated. ${ }^{8}$ Fall in intraoperative PTH level of $>50 \%$ from maximum baseline confirmed a parathyroid adenoma in the current study, despite the ambiguity in frozen section report. Final diagnosis was based on examination of paraffin block.

\section{Conclusion}

In summary, PLA are rare variants of parathyroid adenoma that pose difficulties on preoperative localization and pathologic evaluation especially frozen section. Awareness of this variant, adherence to "basics" of parathyroid gland assessment (weight, measurement, and good frozen section technique) along with intra-operative PTH measurement facilitate recognition during intraoperative setting.

\section{Acknowledgements}

None.

\section{Conflict of interest}

The author declares no conflict of interest.

\section{References}

1. Carlson D. Parathyroid pathology: hyperparathyroidism and parathyroid tumors. Arch Pathol Lab Med. 2010;134:1639-1644.

2. Chow LT, Metreweli C, King WW, et al. Histological changes of parathyroid adenoma after percutaneous injection of ethanol. Histopathology. 1997;30:87-89.

3. Seethala RR, Ogilvie JB, Carty SE. Parathyroid lipoadenomas and lipohyperplasias: clinicopathologic correlations. Am J Surg Pathol. 2008;32:1854-1867.

4. Chow LS, Erickson LA, Abu-Lebdeh HS, et al. Parathyroid lipoadenomas: a rare cause of primary hyperparathyroidism. Endocr Pract. 2006;12:131136.

5. Bansal R, Trivedi P, Sarin J, et al. Lipoadenoma of the parathyroid gland-a rare cause of hyperparathyroidism. Gulf J Oncol. 2012;1:63-65.

6. Ogrin C. A rare case of double parathyroid lipoadenoma with hyperparathyroidism. Am J Med Sci. 2013;346:432-434.

7. Yoon V, Treat K, Maalouf NM. Ectopic atypical parathyroid lipoadenoma: a rare cause of severe primary hyperparathyroidism. J Bone Miner Metab. 2013;31:595-600

8. Lee AY, Wang BY, Heller KS. Importance of intraoperative parathyroid hormone measurement in the diagnosis of parathyroid lipoadenoma. Head Neck. 2011;33:917-919. 